\title{
TITLE:
}

\section{Arc-disjoint in-trees in directed graphs}

$\operatorname{AUTHOR}(S):$

Kamiyama, Naoyuki; Katoh, Naoki; Takizawa, Atsushi

\section{CITATION:}

Kamiyama, Naoyuki ... [et al]. Arc-disjoint in-trees in directed graphs.

Combinatorica 2009, 29(2): 197-214

ISSUE DATE:

2009-03

URL:

http://hdl.handle.net/2433/123379

\section{RIGHT:}

Copyright (C) 2009 Springer; This is not the published version. Please cite only the published version.; この論文は出版社版でありません。引用の 際には出版社版をご確認ご利用ください。 


\title{
Arc-disjoint In-trees in Directed Graphs
}

\author{
Naoyuki KamiYAma* $^{*} \quad$ Naoki KATOH $^{\dagger} \quad$ Atsushi TAKIZAWA $^{\dagger}$
}

April 12, 2008

\begin{abstract}
Given a directed graph $D=(V, A)$, a set of $d$ specified vertices $S=\left\{s_{1}, \ldots, s_{d}\right\} \subseteq V$ and a function $f: S \rightarrow \mathbb{N}$ where $\mathbb{N}$ denotes the set of natural numbers, we present a necessary and sufficient condition such that there exist $\sum_{i=1}^{d} f\left(s_{i}\right)$ arc-disjoint in-trees denoted by $T_{i, 1}, T_{i, 2}, \ldots, T_{i, f\left(s_{i}\right)}$ for every $i=1, \ldots, d$ such that $T_{i, 1}, \ldots, T_{i, f\left(s_{i}\right)}$ are rooted at $s_{i}$ and each $T_{i, j}$ spans the vertices from which $s_{i}$ is reachable. This generalizes the result of Edmonds [2], i.e., the necessary and sufficient condition that for a directed graph $D=(V, A)$ with a specified vertex $s \in V$, there are $k$ arc-disjoint in-trees rooted at $s$ each of which spans $V$. Furthermore, we extend another characterization of packing in-trees of Edmonds [1] to the one in our case.
\end{abstract}

Mathematics Subject Classification: Primary subject classification code 05C70 "Factorization, matching, covering and packing". Secondary subject classification code 05C40 "Connectivity".

*Department of Architecture and Architectural Engineering, Kyoto University, Kyotodaigaku-Katsura, Nishikyo-ku, Kyoto, 615-8540, Japan. E-mail: is.kamiyama@archi.kyoto-u.ac.jp. Supported by JSPS Research Fellowships for Young Scientists.

${ }^{\dagger}$ Department of Architecture and Architectural Engineering, Kyoto University, Kyotodaigaku-Katsura, Nishikyo-ku, Kyoto, 615-8540, Japan. E-mail: \{naoki, kukure\}@archi.kyoto-u.ac.jp. Supported by the project New Horizons in Computing, Grand-in-Aid for Scientific Research on Priority Areas, MEXT Japan. 


\section{Introduction}

Let $D=(V, A)$ be a directed graph which may have parallel arcs. A vertex $v$ is said to be reachable from a vertex $u$ when there is a path from $u$ to $v$. We denote by $e=u v$ an arc $e$ whose tail and head are $u$ and $v$, respectively. If $e=u v$ has no parallel arc, we may simply write $u v$. For $X, Y \subseteq V$, let $\delta(X, Y ; D)=\{e=u v \in A: u \in X, v \in Y\}$. For $W \subseteq V$, we write $\delta^{+}(W ; D)$ and $\delta^{-}(W ; D)$ instead of $\delta(W, V \backslash W ; D)$ and $\delta(V \backslash W, W ; D)$, respectively. For $W \subseteq V$, let $D[W]$ be the subgraph of $D$ induced by $W$. For $u, v \in V$, we denote by $\lambda(u, v ; D)$ the local arc-connectivity from $u$ to $v$ in $D$, i.e.,

$$
\lambda(u, v ; D)=\min \left\{\left|\delta^{-}(W ; D)\right|: u \notin W, v \in W, W \subseteq V\right\} .
$$

Notice that $\lambda(u, v ; D)$ is equal to the maximum number of arc-disjoint paths from $u$ to $v$ in $D$ by Menger's Theorem (see Corollary $9.1 \mathrm{~b}$ in [6]). In this paper, we will not distinguish between a singleton $\{x\}$ and its element $x$.

Edmonds gave a constructive proof of the following theorem.

Theorem 1.1 ([2]) Given a directed graph $D=(V, A)$ with a specified vertex $s \in V$, there exist $k$ arc-disjoint in-trees rooted at $s$ each of which spans $V$ if and only if $\lambda(v, s ; D) \geq k$ holds for every $v \in V \backslash s$.

Alternative proofs are found in [5, 7]. Furthermore, the following variant of Theorem 1.1 is known. Given a directed graph $D=(V, A)$ with a set of $d$ specified vertices $S=\left\{s_{1}, \ldots, s_{d}\right\} \subseteq V$ and a function $f: S \rightarrow \mathbb{N}$ where $\mathbb{N}$ denotes the set of natural numbers, we use the following notations.

- For each $v \in V, R(v)$ denotes the set of vertices in $S$ which are reachable from $v$. 
- For $i=1, \ldots, d, V_{i}$ denotes the set of vertices in $V$ from which $s_{i}$ is reachable.

- $D^{*}=\left(V^{*}, A^{*}\right)$ is a directed graph obtained from $D$ by adding vertex $s^{*}$ and connecting $s_{i}$ to $s^{*}$ with $f\left(s_{i}\right)$ parallel arcs (see Figure 1).

Moreover, we define $f\left(S^{\prime}\right)=\sum_{s_{i} \in S^{\prime}} f\left(s_{i}\right)$ for each $S^{\prime} \subseteq S$.

Theorem 1.2 (Corollary 53.1a in [6]) Given a directed graph $D=(V, A)$ with a set of $d$ specified vertices $S=\left\{s_{1}, \ldots, s_{d}\right\} \subseteq V$ and a function $f: S \rightarrow \mathbb{N}$, there exist $f(S)$ arc-disjoint in-trees denoted by $T_{i, 1}^{\prime}, \ldots, T_{i, f\left(s_{i}\right)}^{\prime}$ for every $i=1, \ldots, d$ such that $T_{i, 1}^{\prime}, \ldots, T_{i, f\left(s_{i}\right)}^{\prime}$ are rooted at $s_{i}$ and each $T_{i, j}^{\prime}$ spans $V$ if and only if $\lambda\left(v, s^{*} ; D^{*}\right) \geq f(S)$ holds for every $v \in V$.

In this paper, we generalize these theorems as follows. Given a set of $d$ specified vertices $S=\left\{s_{1}, \ldots, s_{d}\right\} \subseteq V$ and a function $f: S \rightarrow \mathbb{N}$, we will present a necessary and sufficient condition that there exist $f(S)$ arc-disjoint in-trees denoted by $T_{i, 1}, T_{i, 2}, \ldots, T_{i, f\left(s_{i}\right)}$ for every $i=1, \ldots, d$ such that $T_{i, 1}, \ldots, T_{i, f\left(s_{i}\right)}$ are rooted at $s_{i}$ and each $T_{i, j}$ spans $V_{i}$.

For example, given a directed graph $D$ in Figure $1\left(\right.$ a) with $S=\left\{s_{1}, s_{2}, s_{3}\right\}$ and $f\left(s_{1}\right)=$ 2, $f\left(s_{2}\right)=1, f\left(s_{3}\right)=1$, the set of vertices from which $s_{i}$ is reachable is equal to $\left\{u, v, w, s_{1}\right\}$, the set of vertices from which $s_{2}$ is reachable is equal to $\left\{u, v, w, s_{1}, s_{2}\right\}$, and the set of vertices from which $s_{3}$ is reachable is equal to $\left\{u, v, w, x, y, s_{3}\right\}$. We can see that $T_{1,1}, T_{1,2}, T_{2,1}$, and $T_{3,1}$ shown in Figure 2 are arc-disjoint, and span the vertices from which $s_{1}, s_{2}$ and $s_{3}$ are reachable, respectively.

The main theorem which we will prove in this paper is described as follows.

Theorem 1.3 Given a directed graph $D=(V, A)$ with a set of $d$ specified vertices $S=\left\{s_{1}, \ldots, s_{d}\right\} \subseteq$ $V$ and a function $f: S \rightarrow \mathbb{N}$, there exist $f(S)$ arc-disjoint in-trees denoted by $T_{i, 1}, \ldots, T_{i, f\left(s_{i}\right)}$ for every $i=1, \ldots, d$ such that $T_{i, 1}, \ldots, T_{i, f\left(s_{i}\right)}$ are rooted at $s_{i}$ and each $T_{i, j}$ spans $V_{i}$ if and only if $\lambda\left(v, s^{*} ; D^{*}\right) \geq f(R(v))$ holds for every $v \in V$. 


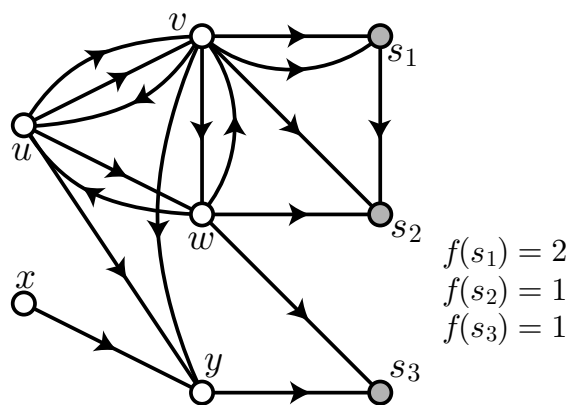

(a)

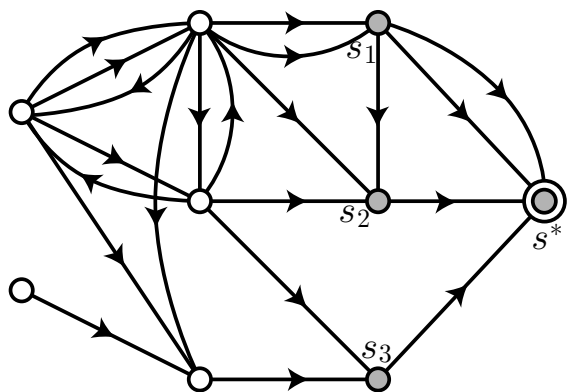

(b)

Figure 1: (a) Directed graph $D$ and function $f$. (b) Transformed graph $D^{*}$.

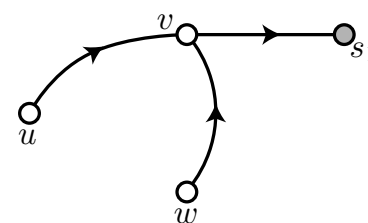

(a)

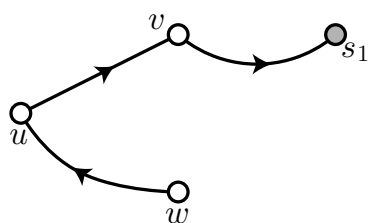

(b)

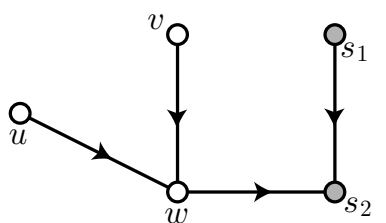

(c)

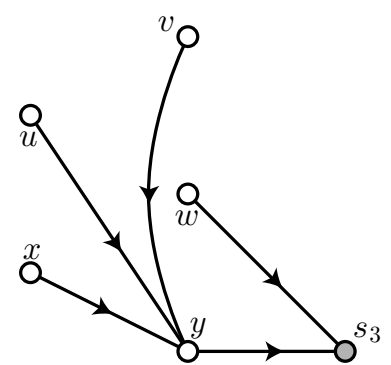

(d)

Figure 2: (a) $T_{1,1}$. (b) $T_{1,2}$. (c) $T_{2,1}$. (d) $T_{3,1}$.

It apparently seems that Theorem 1.3 can be directly derived from Theorem 1.2 by transforming a directed graph $D=(V, A)$ by adding $f\left(s_{i}\right)$ arcs from every vertex not in $V_{i}$ to $s_{i}$. But this is not the case. To see this, let us consider a directed graph $D=(V, A)$ in Figure 3(a) with $S=\left\{s_{1}, s_{2}\right\}$ and $f\left(s_{1}\right)=1, f\left(s_{2}\right)=1$, where $V_{1}=\left\{u, v, w, s_{1}\right\}$ and $V_{2}=\left\{u, v, x, s_{2}\right\}$ hold. Now we add $\operatorname{arcs} x s_{1}, s_{2} s_{1}, w s_{2}$, and $s_{1} s_{2}$ to $A$ so that $R(v)=S$ holds for every $v \in V$ (see Figure $3(\mathrm{~b})$ ). Let $D^{\prime}$ be the resulting graph. From Theorem 1.2, there exist two arc-disjoint in-trees in $D^{\prime}$ denoted by $T_{1,1}^{\prime}$ and $T_{2,1}^{\prime}$ which span $V$, and are rooted at $s_{1}$ and $s_{2}$, respectively. However, removing arcs that are added to obtain $D^{\prime}$ does not always produce the desired $T_{1,1}$ and $T_{2,1}$ such that $T_{1,1}$ is rooted at $s_{1}$ and spans $V_{1}$, and $T_{2,1}$ is rooted at $s_{2}$ and spans $V_{2}$. For $T_{1,1}^{\prime}$ and $T_{2,1}^{\prime}$ which are respectively illustrated in the left side and the right side of Figure 4(a), $T_{1,1}$ and $T_{2,1}$ obtained from $T_{1,1}^{\prime}$ and $T_{2,1}^{\prime}$ by simply removing arcs added to $D$ (dotted arcs) satisfy the statement of Theorem 1.3. However, it is not the case as is seen from Figure 4(b) for 
$T_{1,1}^{\prime}$ and $T_{2,1}^{\prime}$ which are respectively in the left side and the right side of Figure 4(b). Therefore, we can see that Theorem 1.3 can not be immediately derived from Theorem 1.2.

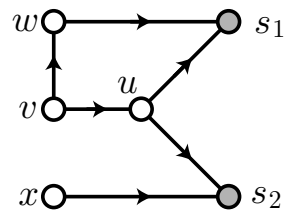

(a)

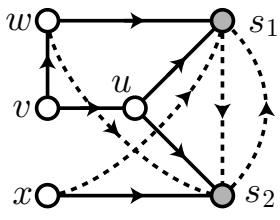

(b)

Figure 3: (a) Input directed graph $D$. (b) Transformed graph $D^{\prime}$.

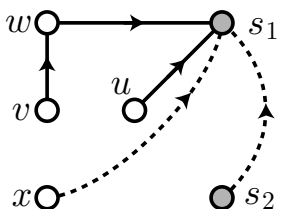

(a)

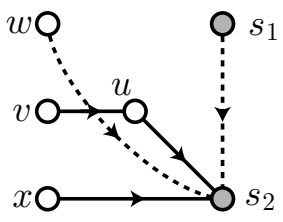$$
\text { ) }
$$

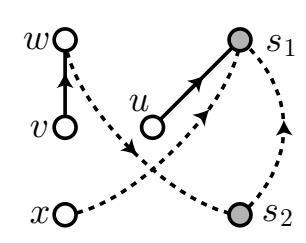

(b)

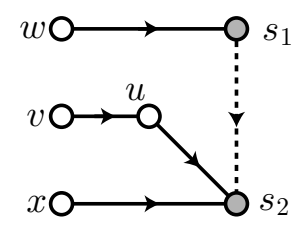

Figure 4: (a) Arc disjoint in-trees $T_{1,1}^{\prime}$ and $T_{2,1}^{\prime}$ for which removing arcs added (dotted arcs) results in $T_{1,1}$ and $T_{2,1}$ that satisfy the statement of Theorem 1.3. (b) Arc disjoint in-trees $T_{1,1}^{\prime}$ and $T_{2,1}^{\prime}$ for which removing arcs added results in $T_{1,1}$ and $T_{2,1}$ that do not satisfy the statement of Theorem 1.3.

In our recent paper [4], we considered the evacuation problem defined on dynamic network and showed that this problem can be efficiently solved if the following property holds for the underlying acyclic graph $D^{\prime}=\left(V^{\prime}, A^{\prime}\right)$ and a sink $s^{\prime} \in V^{\prime}$ of a given dynamic network.

For $P=\left\{s_{1}, \ldots, s_{d}\right\}$ which is the set of vertices in $V^{\prime}$ incident to $s^{\prime}$, there exists $\left|\delta^{-}\left(s^{\prime} ; D^{\prime}\right)\right|$ arc-disjoint in-trees denoted by $T_{i, j}, \ldots, T_{i,\left|\delta\left(s_{i}, s^{\prime} ; D^{\prime}\right)\right|}$ for every $i=1, \ldots, d$ such that $T_{i, j}, \ldots, T_{i,\left|\delta\left(s_{i}, s^{\prime} ; D\right)\right|}$ are rooted at $s_{i}$ and each $T_{i, j}$ spans the vertices from which $s_{i}$ is reachable.

This statement is the same as Theorem 1.3 by setting $D=D^{\prime} \backslash s^{\prime}, S=P$, and $f\left(s_{i}\right)=$ $\left|\delta\left(s_{i}, s^{\prime} ; D^{\prime}\right)\right|$ for $s_{i} \in P$ where $D^{\prime} \backslash s^{\prime}$ denotes the directed graph obtained by removing $s^{\prime}$ and arcs incident to $s^{\prime}$ from $D^{\prime}$. In [4], we proved Theorem 1.3 only for the case where $D$ is acyclic. In this paper, we extend the result in [4] to the case where $D^{\prime}$ is allowed to have cycles. 
This paper is organized as follows. Section 2 gives the proof of Theorem 1.3. In Section 3, we extend another characterization of packing in-trees of Edmonds [1] to the one in our case by using Theorem 1.3.

\section{Proof of Theorem 1.3}

It is not difficult to see that "only if-part" holds. We then prove the "if-part". That is, we assume that for every $v \in V$

$$
\lambda\left(v, s^{*} ; D^{*}\right) \geq f(R(v)) .
$$

We prove the theorem by induction on $f(S)$. In the case of $f(S)=1$, the theorem clearly holds by $|S|=1$.

Assuming that Theorem 1.3 holds for $f(S)=l \geq 1$, we consider the case of $f(S)=l+1$. Let us fix $i \in\{1, \ldots, d\}$ and $e_{i} \in \delta\left(s_{i}, s^{*} ; D^{*}\right)$. To prove the theorem by induction on $f(S)$, we will find an in-tree in $D^{*}$ denoted by $T=(W, B)$ with $W \subseteq V_{i} \cup s^{*}$ such that $T$ is rooted at $s^{*}$ and satisfies (F0) and (F1).

(F0) $\delta^{-}\left(s^{*} ; T\right)=\left\{e_{i}\right\}$, i.e., $T$ has only one $\operatorname{arc} e_{i}$ incident to $s^{*}$.

(F1) For every $v \in V$,

$$
\lambda\left(v, s^{*} ; D^{*} \backslash B\right) \geq \begin{cases}f(R(v))-1, & \text { if } v \in V_{i}, \\ f(R(v)), & \text { if } v \in V \backslash V_{i},\end{cases}
$$

where $D \backslash A^{\prime}$ denotes the directed graph obtained by removing $A^{\prime}$ from $D$, i.e., $D \backslash A^{\prime}=\left(V, A \backslash A^{\prime}\right)$ for each $A^{\prime} \subseteq A$.

If we can find an in-tree $T$ rooted at $s^{*}$ which spans $V_{i}$ and satisfies (F0) and (F1), $T\left[V_{i}\right]$ is an in-tree rooted at $s_{i}$ since a path from every $v \in V_{i}$ to $s^{*}$ in $T$ contains $s_{i}$ from (F0). Moreover, 
since $T$ does not contain any $\operatorname{arc} e=s_{j} s^{*}$ for $j \neq i$ from (F0),

$$
\left|\delta\left(s_{j}, s^{*} ; D^{*} \backslash B\right)\right|= \begin{cases}f\left(s_{j}\right)-1, & \text { if } j=i, \\ f\left(s_{j}\right), & \text { if } j \neq i .\end{cases}
$$

Hence we can regard $D^{*} \backslash B$ as $D^{*}$ for the case of $f(S)=l$, and thus the proof is done.

Here we remark that, in general, every in-tree rooted at $s^{*}$ which spans $V_{i}$ dose not satisfy (F0) and (F1). For example, an in-tree in Figure 5(b) satisfies (F0) and (F1) in a directed graph $D^{*}$ in Figure 5(a) and spans $V_{1}$, while an in-tree in Figure $5(\mathrm{c})$ denoted by $T=(W, B)$ does not satisfy $(\mathrm{F} 1)$ since $\lambda\left(v, s^{*} ; D^{*} \backslash B\right)=0$ holds.

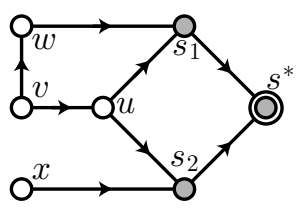

(a)

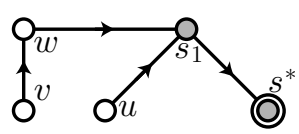

(b)

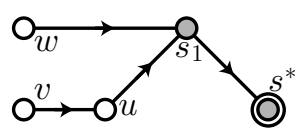

(c)

Figure 5: (a) $D^{*}$ with $S=\left\{s_{1}, s_{2}\right\}$ and $f\left(s_{1}\right)=1, f\left(s_{2}\right)=1$. (b) Feasible in-tree. (c) Infeasible in-tree.

We call an in-tree $T=(W, B)$ with $W \subseteq V_{i} \cup s^{*}$ feasible if $T$ is rooted at $s^{*}$ and satisfies (F0) and (F1). For a feasible in-tree $T=(W, B)$, we call an arc $e=x y$ eligible when $e$ satisfies

(E0) $x \in V_{i} \backslash W$ and $y \in W$,

(E1) $T^{\prime}=(W \cup x, B \cup e)$ is feasible.

That is, if there exists an eligible $\operatorname{arc} e$ for a feasible in-tree $T$, we can extend $T$ by adding $e$ while maintaining feasibility.

We will prove the existence of a feasible in-tree $T$ which spans $V_{i}$ by induction on the number of vertices of $T$. First we prove that for the basis of induction that $T=\left(\left\{s^{*}, s_{i}\right\}, e_{i}\right)$ is feasible. Then, we prove that for any feasible in-tree which does not $\operatorname{span} V_{i}$ there always exists an eligible 
arc. For this, we introduce the notion of a critical set which is a vertex set containing $s^{*}$ such that any arc entering the critical set is not eligible.

Our proof that we can construct a feasible in-tree that spans $V_{i}$ is based on the proof of Theorem 1.1 of Lovász [5]. However, recall that in Theorem 1.1, the local arc-connectivity from every $v \in V \backslash s$ to $s$ is assumed to be at least a constant $k$ which does not depend on $v$. Thus, given an in-tree $T=(W, B)$ rooted at $s$ such that $\lambda(v, s ; D \backslash B) \geq k-1$ holds and $T$ does not span $V$, we can determine whether an arc $e$ can be added to $T$ while maintaining $\lambda(v, s ; D \backslash(B \cup e)) \geq k-1$ for every $v \in V \backslash s$ by simply testing whether $\left|\delta^{-}\left(V^{\prime} ; D \backslash(B \cup e)\right)\right|$ is at least $k-1$ for every $V^{\prime} \subsetneq V$ with $s \in V^{\prime}$. But in our case, the condition of the local arc-connectivity from each $v \in V$ to $s^{*}$ in $D^{*}$ is not uniform. Hence, given a feasible in-tree $T=(W, B)$ which does not span $V_{i}$, to determine whether an arc $e$ is eligible, we have to test whether $\left|\delta^{-}\left(V^{\prime} ; D^{*} \backslash(B \cup e)\right)\right|$ is at least $\min \left\{f(R(v))-1: v \in V_{i} \backslash V^{\prime}\right\}$ and $\min \left\{f(R(v)): v \in V \backslash\left(V_{i} \cup V^{\prime}\right)\right\}$ for every $V^{\prime} \subsetneq V^{*}$ with $s^{*} \in V^{\prime}$. This makes the proof of Theorem 1.3 much harder. To cope with this hardness, we prove that if we choose an appropriate set $X_{\max }, f(R(v))$ takes the same value for every vertex $v \in V_{i} \backslash\left(X_{\max } \cup W\right)$. This clearly holds for the case of Theorem 1.1 and Theorem 1.2. However it is not trivial in our case.

\subsection{Construction of feasible in-tree}

The following inequality can be derived from the definition of (1), which is frequently used in the subsequent discussion. For every $u, v \in V$ and $W \subseteq V$ with $u \notin W$ and $v \in W$,

$$
\lambda(u, v ; D) \leq\left|\delta^{-}(W ; D)\right|
$$

We first prove the following lemma. 
Lemma 2.1 For every subset $A^{\prime}$ of arcs in $D^{*}\left[V_{i} \cup s^{*}\right]$ and $v \in V \backslash V_{i}, \lambda\left(v, s^{*} ; D^{*} \backslash A^{\prime}\right)=$ $\lambda\left(v, s^{*} ; D^{*}\right)$ holds. That is, the local arc-connectivity from $v$ to $s^{*}$ does not change by removing arcs in $A^{\prime}$ from $D^{*}$.

Proof. In $D^{*}$, any path from $v \in V \backslash V_{i}$ to $s^{*}$ does not pass through any vertex in $V_{i}$ from the definition of $V_{i}$. Thus, removing arcs of $D^{*}\left[V_{i} \cup s^{*}\right]$ does not reduce the local arc-connectivity from $v \in V \backslash V_{i}$ to $s^{*}$.

Now we will prove that there exists a feasible in-tree $T=(W, B)$ which spans $V_{i}$ by induction on $|W|$. For the basis of induction, it holds that $T$ is feasible for $T=\left(\left\{s^{*}, s_{i}\right\}, e_{i}\right)$ from Lemma 2.1.

Suppose that we have a feasible in-tree $T=(W, B)$ which does not span $V_{i}$. Then, we will prove that there always exists an eligible arc for $T$. Since $T$ has to satisfy (F0), an arc whose head is $s^{*}$ is not eligible. Furthermore, since $\delta\left(V \backslash V_{i}, V_{i} ; D^{*}\right)=\emptyset$ follows from the definition of $V_{i}$ and $W \backslash s^{*} \subseteq V_{i}$ holds, we have for every $e=x y \in \delta^{-}\left(W \backslash s^{*} ; D^{*} \backslash B\right)$

$$
x \in V_{i} \backslash W
$$

i.e., every $e \in \delta^{-}\left(W \backslash s^{*} ; D^{*} \backslash B\right)$ satisfies (E0). Thus, to prove that there exists an eligible arc for $T$, it is sufficient to prove that there exists an arc $e=x y \in \delta^{-}\left(W \backslash s^{*} ; D^{*} \backslash B\right)$ such that $T^{\prime}=(W \cup x, B \cup e)$ satisfies $(\mathrm{F} 1)$.

It is obvious that $\delta^{-}\left(W \backslash s^{*} ; D^{*} \backslash B\right) \neq \emptyset$ since $T$ does not span $V_{i}$ and $s_{i}$ is reachable from every $v \in V_{i} \backslash W$ in $D$. However, in general, every arc in $\delta^{-}\left(W \backslash s^{*} ; D^{*} \backslash B\right)$ is not eligible. Consider the case where there exists $v \in V_{i}$ with $\lambda\left(v, s^{*} ; D^{*} \backslash B\right)=f(R(v))-1$. In this case, from (1), there must exist $X \subseteq V^{*}$ with $s^{*} \in X, v \notin X$, and $\left|\delta^{-}\left(X ; D^{*} \backslash B\right)\right|=f(R(v))-1$, i.e., $\delta^{-}\left(X ; D^{*} \backslash B\right)$ is a minimum $v-s^{*}$ cut in $D^{*} \backslash B$. Then, an arc $e=x y \in \delta^{-}\left(W \backslash s^{*} ; D^{*} \backslash B\right)$ 
such that $e \in \delta^{-}\left(X ; D^{*} \backslash B\right)$ is not eligible since $T^{\prime}=(W \cup x, B \cup e)$ violates (F1) for $v$.

Here we give the precise description of the above discussion. A vertex set $X \subseteq V^{*}$ with $s^{*} \in X$ is called critical when $X$ satisfies the following conditions.

(C0) $V_{i} \backslash(X \cup W) \neq \emptyset$.

(C1) $\left|\delta^{-}\left(X ; D^{*} \backslash B\right)\right|=f(R(v))-1$ for some $v \in V_{i} \backslash X$.

Lemma 2.2 An arc $e=x y \in \delta^{-}\left(W \backslash s^{*} ; D^{*} \backslash B\right)$ is eligible if there exists no critical set $X \subseteq V^{*}$ with $e \in \delta^{-}\left(X ; D^{*} \backslash B\right)$.

Proof. It is sufficient to prove that $T^{\prime}=(W \cup x, B \cup e)$ satisfies (F1). Suppose that for an arc $e=x y$ that satisfies the lemma assumption, $T^{\prime}$ does not satisfy (F1). Since from Lemma 2.1 the local arc-connectivity from every $w \in V \backslash V_{i}$ to $s^{*}$ does not change by removing arcs in $D\left[V_{i}\right]$ (notice that $e$ is an arc in $D\left[V_{i}\right]$ from (4)), there exists $v \in V_{i}$ such that $\lambda\left(v, s^{*} ; D^{*} \backslash(B \cup e)\right) \leq$ $f(R(v))-2$. From (1), there exists $Y \subseteq V^{*}$ with $s^{*} \in Y$ and $v \notin Y$ such that

$$
\left|\delta^{-}\left(Y ; D^{*} \backslash(B \cup e)\right)\right| \leq f(R(v))-2
$$

We will show that $Y$ satisfies $(\mathrm{C} 0)$ and $(\mathrm{C} 1)$, and $e \in \delta^{-}\left(Y ; D^{*} \backslash B\right)$ holds, which contradicts that $e$ satisfies the lemma assumption.

Since $T$ satisfies (F1), $\left|\delta^{-}\left(Y ; D^{*} \backslash B\right)\right| \geq f(R(v))-1$ follows from (3). Thus, since $\mid \delta^{-}\left(Y ; D^{*} \backslash\right.$ $B)|-| \delta^{-}\left(Y ; D^{*} \backslash(B \cup e)\right) \mid$ is at most one, $\left|\delta^{-}\left(Y ; D^{*} \backslash B\right)\right|$ must be equal to $f(R(v))-1$ (i.e., $Y$ satisfies $(\mathrm{C} 1))$ and $e \in \delta^{-}\left(Y ; D^{*} \backslash B\right)$ holds by (5).

Since $x \in V_{i} \backslash W$ follows from (4) and $x \notin Y$ follows from $e \in \delta^{-}\left(Y ; D^{*} \backslash B\right), x \in V_{i} \backslash(Y \cup W)$ holds. Thus, $Y$ satisfies (C0). This completes the proof.

We now consider the case where there exists a critical set. From now on, we prove that in 
this case, there always exists an eligible arc $e \in \delta^{-}\left(W \backslash s^{*} ; D^{*} \backslash B\right)$, too. To prove this, let us fix $X_{\max }$ as a critical set which satisfies

$$
\left|X_{\max }\right|=\max \{|X|: X \text { is critical }\}
$$

and let $v_{\max } \in V_{i} \backslash X_{\max }$ be a vertex satisfying (C1) for $X_{\max }$, i.e., $v_{\max }$ satisfies

$$
\left|\delta^{-}\left(X_{\max } ; D^{*} \backslash B\right)\right|=f\left(R\left(v_{\max }\right)\right)-1 .
$$

From (1) and (F1),

$$
\lambda\left(v_{\max }, s^{*} ; D^{*} \backslash B\right)=f\left(R\left(v_{\max }\right)\right)-1 .
$$

The following lemma concerning $X_{\max }$ and $v_{\max }$ plays a crucial role in our proof.

Lemma 2.3 For $X_{\max }$ and $v_{\max }$ defined above, $f(R(w))=f\left(R\left(v_{\max }\right)\right)$ holds for every $w \in$ $V_{i} \backslash\left(X_{\max } \cup W\right)$

Since the proof of Lemma 2.3 is long, we prove the theorem by using this lemma before giving its proof. The proof of this lemma is given in the following subsection.

First we prove the following lemma.

Lemma 2.4 There exists an arc $e=x y$ with $x \in V_{i} \backslash\left(X_{\max } \cup W\right)$ and $y \in W \backslash X_{\max }$ in $D^{*} \backslash B$.

Proof. Since both of a tail and a head of every arc in $B$ are contained in $W$,

$$
\delta^{-}\left(X_{\max } \cup W ; D^{*} \backslash B\right)=\delta^{-}\left(X_{\max } \cup W ; D^{*}\right) .
$$

Next we prove

$$
\left|\delta^{-}\left(X_{\max } \cup W ; D^{*}\right)\right| \geq f\left(R\left(v_{\max }\right)\right) .
$$


From (C0), there exists $w \in V_{i} \backslash\left(X_{\max } \cup W\right)$. From (3), $w \notin X_{\max } \cup W$ and (2),

$$
\left|\delta^{-}\left(X_{\max } \cup W ; D^{*}\right)\right| \underbrace{\geq \lambda\left(w, s^{*} ; D^{*}\right)}_{\text {from }(3)} \underbrace{\geq f(R(w))}_{\text {from }(2)} .
$$

Thus, (10) follows from Lemma 2.3. Hence,

$$
\begin{aligned}
\left|\delta^{-}\left(X_{\max } \cup W ; D^{*} \backslash B\right)\right| & =\left|\delta^{-}\left(X_{\max } \cup W ; D^{*}\right)\right| \quad(\text { from }(9)) \\
& \geq f\left(R\left(v_{\max }\right)\right) \quad(\text { from }(10)) \\
& >\left|\delta^{-}\left(X_{\max } ; D^{*} \backslash B\right)\right| \quad(\text { from }(7)) .
\end{aligned}
$$

From this inequality, we can see that there exists at least one arc $e=x y$ with $x \in V^{*} \backslash\left(X_{\max } \cup W\right)$ and $y \in W \backslash X_{\max }$. Hence, the lemma holds since $x \in V_{i} \backslash W$ follows from (4).

Let an arc satisfying Lemma 2.4 be $\hat{e}=\hat{x} \hat{y}$ with $\hat{x} \in V_{i} \backslash\left(X_{\max } \cup W\right)$ and $\hat{y} \in W \backslash X_{\max }$ (see Figure 6). From Lemma 2.2, if there exists no critical set $Y$ such that $\hat{e} \in \delta^{-}\left(Y ; D^{*} \backslash B\right)$, $\hat{e}$ is eligible.

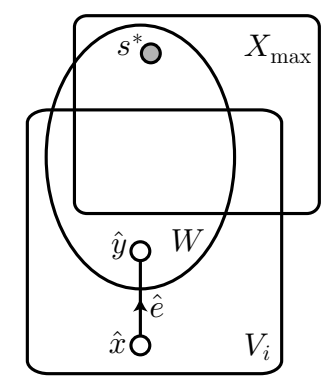

Figure 6: Illustration of $\hat{e}$.

Lemma 2.5 There exists no critical set $Y \subseteq V^{*}$ such that $\hat{e}=\hat{x} \hat{y} \in \delta^{-}\left(Y ; D^{*} \backslash B\right)$.

Proof. We will show that if there exists such $Y, X_{\max } \cup Y$ is critical and $\hat{x}$ satisfies (C1) for 
$X_{\max } \cup Y$. This implies that $\left|X_{\max }\right|<\left|X_{\max } \cup Y\right|$ holds since $\hat{y} \in Y \backslash X_{\max }$ follows from $\hat{y} \in W \backslash X_{\max }$ and $\hat{y} \in Y$, which contradicts the maximality of $X_{\max }$ in (6).

From $\hat{e} \in \delta^{-}\left(Y ; D^{*} \backslash B\right), \hat{x} \notin Y$ holds. Thus, $\hat{x} \in V_{i} \backslash\left(X_{\max } \cup Y \cup W\right)$ holds since $\hat{x} \in$ $V_{i} \backslash\left(X_{\max } \cup W\right)$ follows from the definition of $\hat{e}$. Hence, $X_{\max } \cup Y$ satisfies $(\mathrm{C} 0)$ for $X=X_{\max } \cup Y$.

What remains is to prove that $\left|\delta^{-}\left(X_{\max } \cup Y ; D^{*} \backslash B\right)\right|=f(R(\hat{x}))-1$, i.e., (C1) holds. From $\hat{x} \notin X_{\max } \cup Y,(3)$ and $(\mathrm{F} 1)$,

$$
\left|\delta^{-}\left(X_{\max } \cup Y ; D^{*} \backslash B\right)\right| \geq \underbrace{\geq \lambda\left(\hat{x}, s^{*} ; D^{*} \backslash B\right)}_{\text {from (3) }} \underbrace{f(R(\hat{x}))-1}_{\text {from (F1) }} .
$$

Thus, to prove that $(\mathrm{C} 1)$ holds, it is sufficient to show

$$
\left|\delta^{-}\left(X_{\max } \cup Y ; D^{*} \backslash B\right)\right| \leq f(R(\hat{x}))-1
$$

Since $Y$ is critical, there exists $w_{\mathrm{cr}} \in V_{i} \backslash Y$ satisfying (C1) for $Y$, i.e,

$$
\left|\delta^{-}\left(Y ; D^{*} \backslash B\right)\right|=f\left(R\left(w_{\mathrm{cr}}\right)\right)-1 .
$$

Then, from the submodularity of $\left|\delta^{-}\left(\cdot ; D^{*} \backslash B\right)\right|$,

$$
\begin{aligned}
f\left(R\left(v_{\max }\right)\right)-1+f\left(R\left(w_{\mathrm{cr}}\right)\right)-1 & =\left|\delta^{-}\left(X_{\max } ; D^{*} \backslash B\right)\right|+\left|\delta^{-}\left(Y ; D^{*} \backslash B\right)\right| \quad(\text { by }(7) \text { and (13)) } \\
& \geq\left|\delta^{-}\left(X_{\max } \cap Y ; D^{*} \backslash B\right)\right|+\left|\delta^{-}\left(X_{\max } \cup Y ; D^{*} \backslash B\right)\right| .
\end{aligned}
$$


Since $v_{\max }, w_{\text {cr }} \notin X_{\max } \cap Y$ follows from $v_{\max } \notin X_{\max }$ and $w_{\text {cr }} \notin Y$, we have

$$
\begin{aligned}
\left|\delta^{-}\left(X_{\max } \cap Y ; D^{*} \backslash B\right)\right| & \geq \max \left\{\lambda\left(v_{\max }, s^{*} ; D^{*} \backslash B\right), \lambda\left(w_{\mathrm{cr}}, s^{*} ; D^{*} \backslash B\right)\right\} \quad(\text { from }(3)) \\
& \geq \max \left\{f\left(R\left(v_{\max }\right)\right), f\left(R\left(w_{\mathrm{cr}}\right)\right)\right\}-1 \quad(\text { from }(\mathrm{F} 1)) .
\end{aligned}
$$

In the case of $f\left(R\left(w_{\mathrm{cr}}\right)\right) \geq f\left(R\left(v_{\max }\right)\right)$, we straightforwardly have

$$
\left|\delta^{-}\left(X_{\max } \cup Y ; D^{*} \backslash B\right)\right| \leq f\left(R\left(v_{\max }\right)\right)-1 \quad \text { (from (14) and (15)). }
$$

In the case of $f\left(R\left(w_{\mathrm{cr}}\right)\right)<f\left(R\left(v_{\max }\right)\right)$, we have $\left|\delta^{-}\left(X_{\max } \cup Y ; D^{*} \backslash B\right)\right| \leq f\left(R\left(w_{\mathrm{cr}}\right)\right)-1$ from (14) and (15), and hence (16) follows from $f\left(R\left(w_{\mathrm{cr}}\right)\right)<f\left(R\left(v_{\max }\right)\right)$.

Since $\hat{x} \in V_{i} \backslash\left(X_{\max } \cup W\right)$ from the definition of $\hat{e}, f(R(\hat{x}))=f\left(R\left(v_{\max }\right)\right)$ follows from Lemma 2.3. Thus, (12) follows from (16). This completes the proof.

Proof of Theorem 1.3. Assuming that there exists a feasible in-tree $T=(W, B)$ such that $|W| \geq 2$ and $|W|<\left|V_{i}\right|$, we will prove that there exists a feasible in-tree $T^{\prime}=\left(W^{\prime}, B^{\prime}\right)$ such that $\left|W^{\prime}\right|=|W|+1$, i.e., there exists an eligible $\operatorname{arc}$ for $T$. If there exists no critical set, it follows from Lemma 2.2 that any $e \in \delta^{-}\left(W \backslash s^{*}, D^{*} \backslash B\right)$ is eligible. Otherwise, letting $X_{\max }$ be a critical set satisfying (6), we can see from Lemmas 2.4 and 2.5 that there exists an eligible arc $e=x y$ with $x \in V_{i} \backslash\left(X_{\max } \cup W\right)$ and $y \in W \backslash X_{\max }$. Hence, repeating this process, we eventually have a feasible in-tree $T=(W, B)$ which spans $V_{i}$. This completes the proof.

\subsection{Proof of Lemma 2.3}

In this subsection, we prove Lemma 2.3.

From the definition of a feasible in-tree, $\lambda\left(w, s^{*} ; D^{*} \backslash B\right)$ is at least $f(R(w))-1$ for every $w \in V_{i}$. However, we can see from the following lemma that in fact, $\lambda\left(w, s^{*} ; D^{*} \backslash B\right)$ is equal to 
$f(R(w))-1$ for every $w \in V_{i}$.

Lemma 2.6 For every $w \in V_{i}, \lambda\left(w, s^{*} ; D^{*} \backslash B\right)=f(R(w))-1$ holds.

Proof. From the way of construction of $D^{*}$ and the definition of $R(\cdot)$, every set of $f(R(w))$ arcdisjoint paths from $w$ to $s^{*}$ in $D^{*}$ use all $\operatorname{arcs}$ in $\delta\left(R(w), s^{*} ; D^{*}\right)$. From $(\mathrm{F} 0), \mid \delta\left(R(w), s^{*} ; D^{*} \backslash\right.$ $B) \mid=f(R(w))-1$ follows. Thus, $\lambda\left(w, s^{*} ; D^{*} \backslash B\right) \leq f(R(w))-1$ holds since $\lambda\left(w, s^{*} ; D^{*} \backslash B\right)$ is equal to the maximum number of arc-disjoint paths from $w$ to $s^{*}$ in $D^{*} \backslash B$. Then, the lemma follows from (F1).

To prove Lemma 2.3, we will prove $\lambda\left(w, s^{*} ; D^{*} \backslash B\right)=\lambda\left(v_{\max }, s^{*} ; D^{*} \backslash B\right)$ for every $w \in$ $V_{i} \backslash\left(X_{\max } \cup W\right)$. For this, we prove some lemmas.

Lemma 2.7 For $X_{\max }$ and $v_{\max }$ defined above, $\lambda\left(w, s^{*} ; D^{*} \backslash B\right) \leq \lambda\left(v_{\max }, s^{*} ; D^{*} \backslash B\right)$ for every $w \in V_{i} \backslash\left(X_{\max } \cup W\right)$.

Proof. Since $w \notin X_{\max }$ follows from $w \in V_{i} \backslash\left(X_{\max } \cup W\right)$,

$$
f\left(R\left(v_{\max }\right)\right)-1 \underbrace{=\left|\delta^{-}\left(X_{\max } ; D^{*} \backslash B\right)\right|}_{\text {from }(7)} \underbrace{\geq \lambda\left(w, s^{*} ; D^{*} \backslash B\right)}_{\text {from }(3)} .
$$

This inequality and (8) imply the lemma.

We will prove by contradiction that there exists no vertex $w \in V_{i} \backslash\left(X_{\max } \cup W\right)$ such that

$$
\lambda\left(w, s^{*} ; D^{*} \backslash B\right)<\lambda\left(v_{\max }, s^{*} ; D^{*} \backslash B\right) .
$$


Assuming that there exists a vertex $\tilde{w} \in V_{i} \backslash\left(X_{\max } \cup W\right)$ satisfying (17), let

$$
\begin{gathered}
U:=\left\{u \in V^{*} \backslash X_{\max }: R(u) \subseteq R(\tilde{w})\right\}, \\
P:=V^{*} \backslash U .
\end{gathered}
$$

Notice that $\tilde{w} \in U$ follows from (18).

Lemma 2.8 For $P$ and $v_{\max }$ defined above, $v_{\max } \in P$ holds.

Proof. It is sufficient to prove $v_{\max } \notin U$ from $v_{\max } \notin X_{\max }$ and the definition of (19). To prove $v_{\max } \notin U$, we will show $R\left(v_{\max }\right) \nsubseteq R(\tilde{w})$ since this implies $v_{\max } \notin U$ from (18). If $R\left(v_{\max }\right) \subseteq R(\tilde{w})$,

$$
f\left(R\left(v_{\max }\right)\right) \leq f(R(\tilde{w}))
$$

follows from the definition of $f(\cdot)$. Hence

$$
\lambda\left(\tilde{w}, s^{*} ; D^{*} \backslash B\right) \underbrace{\geq f(R(\tilde{w}))-1}_{\text {from }(\mathrm{F} 1)} \underbrace{\geq f\left(R\left(v_{\max }\right)\right)-1}_{\text {from }(20)} \underbrace{=\lambda\left(v_{\max }, s^{*} ; D^{*} \backslash B\right)}_{\text {from }(8)} .
$$

This contradicts (17), and completes the proof.

Lemma 2.9 For $P$ and $X_{\max }$ defined above, $\delta^{-}\left(P ; D^{*} \backslash B\right) \subseteq \delta^{-}\left(X_{\max } ; D^{*} \backslash B\right)$ holds.

Proof. From (19), it is sufficient to prove

$$
\delta\left(U, V^{*} \backslash\left(X_{\max } \cup U\right) ; D^{*} \backslash B\right)=\emptyset,
$$

(see Figure 7). If there exists an $\operatorname{arc} e=x y$ in the arc set of the left hand side of (21), $x \in U$ and $y \notin X_{\max } \cup U$ hold. From (18), $x \in U$ is equivalent to $R(x) \subseteq R(\tilde{w})$, and also $y \notin X_{\max } \cup U$ 
is equivalent to $R(y) \nsubseteq R(\tilde{w})$. However, this contradicts $R(y) \subseteq R(x)$ which follows from the definition of $R(\cdot)$. This completes the proof.

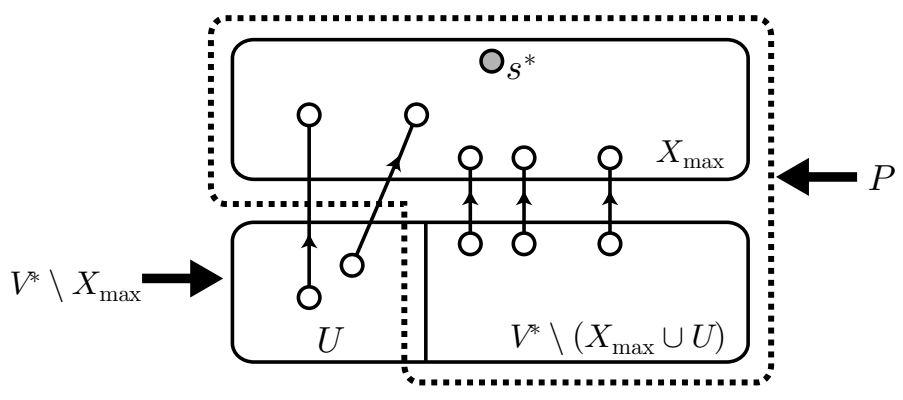

Figure 7: Illustration of (21). There exists no arc whose tail is in $U$ and whose head is in $V^{*} \backslash\left(X_{\max } \cup U\right)$.

Let $H$ be the set of heads of all $\operatorname{arcs}$ in $\delta^{-}\left(P ; D^{*} \backslash B\right)$.

Lemma 2.10 For $\tilde{w}$ and $H$ defined above, $\bigcup_{h \in H} R(h) \subseteq R(\tilde{w})$ holds.

Proof. Assume that there exist $s_{j} \in\left(\bigcup_{h \in H} R(h)\right) \backslash R(\tilde{w})$ and $e=x y \in \delta^{-}\left(P ; D^{*} \backslash B\right)$ such that $s_{j} \in R(y)$. Notice that $e \in \delta^{-}\left(P ; D^{*} \backslash B\right)$ implies $x \notin P$, which implies $x \in U$ by (19). Thus, $R(x) \subseteq R(\tilde{w})$ follows from $x \in U$ and (18). Hence, since $R(y) \subseteq R(x)$ follows from the definition of $R(\cdot), s_{j} \in R(y)$ implies $s_{j} \in R(\tilde{w})$. This contradicts $s_{j} \in\left(\bigcup_{h \in H} R(h)\right) \backslash R(\tilde{w})$.

Lemma 2.11 For $P$ and $\tilde{w}$ defined above, there exist at most $f(R(\tilde{w}))-1$ arc-disjoint paths from $v_{\max }$ to $s^{*}$ in $D^{*} \backslash B$ that use arcs in $\delta^{-}\left(P ; D^{*} \backslash B\right)$.

Proof. Since a path from $v_{\max }$ to $s^{*}$ in $D^{*} \backslash B$ that uses an arc in $\delta^{-}\left(P ; D^{*} \backslash B\right)$ can not pass through $s_{j} \in S \backslash\left(\bigcup_{h \in H} R(h)\right)$ for $H$ defined above, the maximum number of arc-disjoint paths from $v_{\max }$ to $s^{*}$ in $D^{*} \backslash B$ that use $\operatorname{arcs}$ in $\delta^{-}\left(P ; D^{*} \backslash B\right)$ is at most $\left|\delta\left(\bigcup_{h \in H} R(h), s^{*} ; D^{*} \backslash B\right)\right|$. Since $\left(\bigcup_{h \in H} R(h)\right) \subseteq R(\tilde{w})$ follows from Lemma 2.10, $\left|\delta\left(\bigcup_{h \in H} R(h), s^{*} ; D^{*} \backslash B\right)\right| \leq\left|\delta\left(R(\tilde{w}), s^{*} ; D^{*} \backslash B\right)\right|$ holds. Thus, since $\left|\delta\left(R(\tilde{w}), s^{*} ; D^{*} \backslash B\right)\right|=f(R(\tilde{w}))-1$ follows from the definition of $D^{*}$ and 
(F0), the lemma follows (see Figure 8(a)).

Lemma 2.12 For $P$ and $\tilde{w}$ defined above, $\left|\delta^{-}\left(P ; D^{*} \backslash B\right)\right|=f(R(\tilde{w}))-1$ holds.

Proof. Recalling that $\tilde{w} \notin P$ follows from $\tilde{w} \in U$ and (19),

$$
\left|\delta^{-}\left(P ; D^{*} \backslash B\right)\right| \underbrace{\geq \lambda\left(\tilde{w}, s^{*} ; D^{*} \backslash B\right)}_{\text {from }(3)} \underbrace{\geq f(R(\tilde{w}))-1}_{\text {from }(\mathrm{F} 1)} .
$$

Now suppose $\left|\delta^{-}\left(P ; D^{*} \backslash B\right)\right|>f(R(\tilde{w}))-1$. Then,

$$
\begin{aligned}
& \left|\delta^{-}\left(X_{\max } ; D^{*} \backslash B\right) \backslash \delta^{-}\left(P ; D^{*} \backslash B\right)\right| \\
= & \left|\delta^{-}\left(X_{\max } ; D^{*} \backslash B\right)\right|-\left|\delta^{-}\left(P ; D^{*} \backslash B\right)\right| \quad \text { (by Lemma 2.9) } \\
= & \left(f\left(R\left(v_{\max }\right)\right)-1\right)-\left|\delta^{-}\left(P ; D^{*} \backslash B\right)\right| \quad(\text { by }(7)) \\
< & \left(f\left(R\left(v_{\max }\right)\right)-1\right)-(f(R(\tilde{w}))-1) \quad \text { (by the assumption made above) } \\
= & f\left(R\left(v_{\max }\right)\right)-f(R(\tilde{w})) .
\end{aligned}
$$

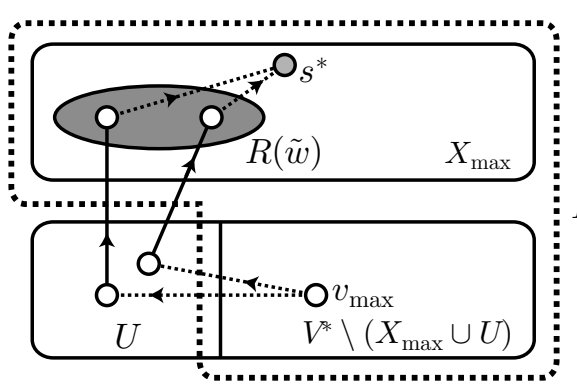

(a)

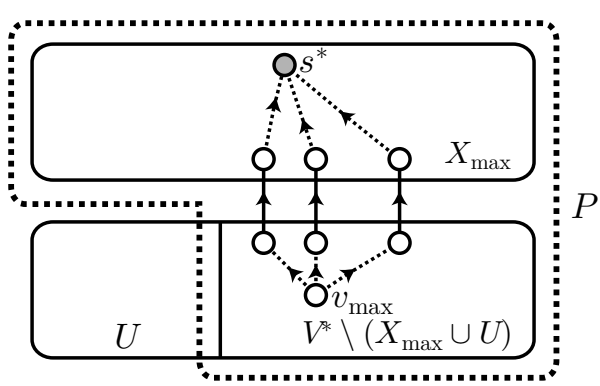

(b)

Figure 8: (a) Arc-disjoint paths that use arcs in $\delta^{-}\left(P ; D^{*} \backslash B\right)$. Bold lines represent arcs in $\delta^{-}\left(P ; D^{*} \backslash B\right)$. (b) Arc-disjoint paths that use arcs in $\delta^{-}\left(X_{\max } ; D^{*} \backslash B\right) \backslash \delta^{-}\left(P ; D^{*} \backslash B\right)$. Bold lines represent arcs in $\delta^{-}\left(X_{\max } ; D^{*} \backslash B\right) \backslash \delta^{-}\left(P ; D^{*} \backslash B\right)$.

Let us consider the maximum number of arc-disjoint paths from $v_{\max }$ to $s^{*}$ in $D^{*} \backslash B$. A 
path from $v_{\max }$ to $s^{*}$ in $D^{*} \backslash B$ uses at least one $\operatorname{arc}$ in $\delta^{-}\left(X_{\max } ; D^{*} \backslash B\right)$. Here we partition $\delta^{-}\left(X_{\max } ; D^{*} \backslash B\right)$ into $\delta^{-}\left(P ; D^{*} \backslash B\right)$ and $\delta^{-}\left(X_{\max } ; D^{*} \backslash B\right) \backslash \delta^{-}\left(P ; D^{*} \backslash B\right)$. The maximum number of arc-disjoint paths from $v_{\max }$ to $s^{*}$ in $D^{*} \backslash B$ that use an $\operatorname{arc}$ in $\delta^{-}\left(P ; D^{*} \backslash B\right)$ is at most $f(R(\tilde{w}))-1$ from Lemma 2.11 (see Figure $8(\mathrm{a})$ ). Furthermore, the maximum number of arc-disjoint paths from $v_{\max }$ to $s^{*}$ in $D^{*} \backslash B$ that use an arc in $\delta^{-}\left(X_{\max } ; D^{*} \backslash B\right) \backslash \delta^{-}\left(P ; D^{*} \backslash B\right)$ is at most $f\left(R\left(v_{\max }\right)\right)-f(R(\tilde{w}))-1$ from (22) (see Figure $\left.8(\mathrm{~b})\right)$. This implies that there can not exist $f\left(R\left(v_{\max }\right)\right)-1$ arc-disjoint paths from $v_{\max }$ to $s^{*}$ in $D^{*} \backslash B$, which contradicts $(\mathrm{F} 1)$. This completes the proof.

We are now ready to prove the following lemma.

Lemma 2.13 For $X_{\max }$ and $v_{\max }$ defined above, $\lambda\left(w, s^{*} ; D^{*} \backslash B\right)=\lambda\left(v_{\max }, s^{*} ; D^{*} \backslash B\right)$ for every $w \in V_{i} \backslash\left(X_{\max } \cup W\right)$.

Proof. For $P$ defined in (19), $\left|X_{\max }\right|<|P|$ holds since $v_{\max } \in P \backslash X_{\max }$ follows from Lemma 2.8 and the definition of $v_{\max }$ and $X_{\max } \subseteq P$ holds by (19).

Here we prove that $P$ is a critical set. From $s^{*} \notin U, s^{*} \in P$ follows. Since $\tilde{w} \notin W$ follows from $\tilde{w} \in V_{i} \backslash\left(X_{\max } \cup W\right)$, and $\tilde{w} \notin P$ follows from $\tilde{w} \notin X_{\max }, \tilde{w} \in U$ and (19), $\tilde{w} \in V_{i} \backslash(P \cup W)$, i.e., $P$ satisfies $(C 0)$. Moreover, from Lemma 2.12 and $\tilde{w} \notin V_{i} \backslash P, P$ satisfies $(\mathrm{C} 1)$ for $\tilde{w}$. This contradicts the maximality of $X_{\max }$ in (6). Thus, the lemma follows from Lemma 2.7 .

Proof of Lemma 2.3. For every $w \in V_{i} \backslash\left(X_{\max } \cup W\right)$,

$$
f(R(w))-1 \underbrace{=\lambda\left(w, s^{*} ; D^{*} \backslash B\right)}_{\text {from Lemma } 2.6} \underbrace{=\lambda\left(v_{\max }, s^{*} ; D^{*} \backslash B\right)}_{\text {from Lemma } 2.13} .
$$

The lemma follows from this equality and (8). 


\section{Another Characterization of Packing In-trees}

Edmonds [1] showed the following another characterization of packing in-trees. For directed graphs $D_{1}=\left(W_{1}, B_{1}\right)$ and $D_{2}=\left(W_{2}, B_{2}\right)$, the union of $D_{1}$ and $D_{2}$ is defined as $D_{3}=\left(W_{1} \cup\right.$ $\left.W_{2}, B_{1} \cup B_{2}\right)$. We call a subgraph $T$ of $D$ a tree when $T$ has no cycle in the graph obtained by ignoring the direction of $\operatorname{arcs}$ of $D$. Here we define a feasible set of $k$ trees $\mathcal{T}$ in $D=(V, A)$ with specified vertex $s \in V$ as a set of $k$ arc-disjoint trees such that each tree spans $V$ and for every $v \in V$

$$
\left|\delta^{+}(v ; F)\right|=\left\{\begin{aligned}
k, & \text { if } v \in V \backslash s, \\
0, & \text { if } v=s
\end{aligned}\right.
$$

where $F$ is the union of the $k$ arc-disjoint trees in $\mathcal{T}$.

Theorem $3.1([\mathbf{1}])$ Given a directed graph $D=(V, A)$ with a specified vertex $s \in V$, there exist $k$ arc-disjoint in-trees rooted at $s$ each of which spans $V$ if and only if there exists a feasible set of $k$ trees.

Gabow [3] presented an efficient algorithm to find arc-disjoint in-trees defined in Theorem 1.1 by using Theorem 3.1. We extend this characterization to the one in our case as follows. Here we define a feasible set of $f(S)$ subtrees $\mathcal{T}^{*}$ in $D^{*}=\left(V^{*}, A^{*}\right)$ as a set of $f(S)$ arc-disjoint trees denoted by $T_{i, 1}^{*}, T_{i, 2}^{*}, \ldots, T_{i, f\left(s_{i}\right)}^{*}$ for every $i=1, \ldots, d$ such that each $T_{i, j}^{*}$ spans $V_{i} \cup s^{*}$ and for every $v \in V$

$$
\left|\delta^{+}\left(v ; F^{*}\right)\right|= \begin{cases}f(R(v)), & \text { if } v \in V, \\ 0, & \text { if } v=s^{*}\end{cases}
$$

where $F^{*}$ is the union of the $f(S)$ arc-disjoint trees in $\mathcal{T}^{*}$. Figures $9(\mathrm{a}),(\mathrm{b}),(\mathrm{c})$, and (d) show $T_{1,1}^{*}, T_{1,2}^{*}, T_{2,1}^{*}$, and $T_{3,1}^{*}$ respectively which compose a feasible set of four subtrees in $D^{*}$ shown in Figure 1(b). 


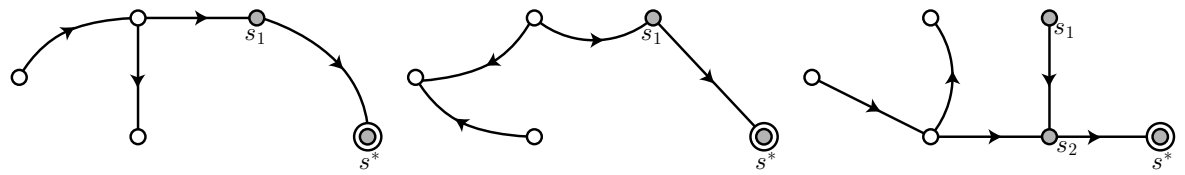

(a) (b)

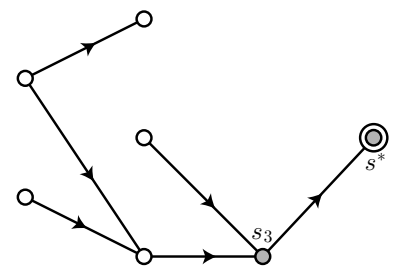

(d)

Figure 9: (a) $T_{1,1}^{*}$. (b) $T_{1,2}^{*}$. (c) $T_{2,1}^{*}$. (d) $T_{3,1}^{*}$.

The proof of the following theorem is based on the proof of Theorem 3.1 of Gabow (see Corollary 2.1 in [3]).

Theorem 3.2 Given a directed graph $D=(V, A)$ with a set of d specified vertices $S=\left\{s_{1}, \ldots, s_{d}\right\} \subseteq$ $V$ and a function $f: S \rightarrow \mathbb{N}$, there exist $f(S)$ arc-disjoint in-trees denoted by $T_{i, 1}, \ldots, T_{i, f\left(s_{i}\right)}$ for every $i=1, \ldots, d$ such that $T_{i, 1}, \ldots, T_{i, f\left(s_{i}\right)}$ are rooted at $s_{i}$ and each $T_{i, j}$ spans $V_{i}$ if and only if there exists a feasible set of $f(S)$ subtrees.

Proof. Only if-part. Since $T_{i, 1}^{*}, T_{i, 2}^{*}, \ldots, T_{i, f\left(s_{i}\right)}^{*}$ for every $i=1, \ldots, d$ which compose a feasible set of $f(S)$ subtrees $\mathcal{T}^{*}$ can be straightforwardly constructed from $T_{i, 1}, T_{i, 2}, \ldots, T_{i, f\left(s_{i}\right)}$ for every $i=1, \ldots, d$ which satisfy the condition of the theorem.

If-part. Suppose that there exist $T_{i, 1}^{*}, \ldots, T_{i, f\left(s_{i}\right)}^{*}$ for every $i=1, \ldots, d$ which compose a feasible set of $f(S)$ subtrees $\mathcal{T}^{*}$. From Theorem 1.3, it is sufficient to prove that $\lambda\left(v, s^{*} ; D^{*}\right) \geq f(R(v))$ holds for every $v \in V$.

From (1), the statement that $\lambda\left(v, s^{*} ; D^{*}\right) \geq f(R(v))$ holds for every $v \in V$ is equivalent to

(i) $\delta^{+}\left(W ; D^{*}\right) \geq f(R(v))$ holds for every $v \in V$ and $W \subseteq V$ with $v \in W$.

Thus, we will prove the statement (i). Let us fix $v \in V$ and $W \subseteq V$ with $v \in W$. Recall that $F^{*}$ is the union of the $f(S)$ arc-disjoint trees in $\mathcal{T}^{*}$. Thus, precisely $\sum_{w \in W} f(R(w)) \operatorname{arcs}$ of $F^{*}$ have their tails in $W$ from the definition of $\mathcal{T}^{*}$ and $s^{*} \notin W$. Here let $I_{W}$ be the set of $i \in\{1, \ldots, d\}$ such that $W \cap V_{i} \neq \emptyset$. Here we consider the sum of the number of $\operatorname{arcs}$ of $T_{i, 1}^{*}, T_{i, 2}^{*}, \ldots, T_{i, f\left(s_{i}\right)}^{*}$ 
which have both ends in $W$ for $i \in I_{W}$. Since $T_{i, j}^{*}$ is a tree and spans $V_{i}$, the number of arcs of $T_{i, j}^{*}$ which have both ends in $W$ is at most $\left|W \cap V_{i}\right|-1$. Thus, at most $\sum_{i \in I_{W}}\left(\left|W \cap V_{i}\right|-1\right) \cdot f\left(s_{i}\right)$ arcs of $F^{*}$ have both ends in $W$. Thus, to prove the statement (i), since

$$
\delta^{+}\left(W ; D^{*}\right) \geq \sum_{w \in W} f(R(w))-\sum_{i \in I_{W}}\left(\left|W \cap V_{i}\right|-1\right) \cdot f\left(s_{i}\right)
$$

follows from the above discussion, it is sufficient to prove

$$
\sum_{w \in W} f(R(w))-\sum_{i \in I_{W}}\left(\left|W \cap V_{i}\right|-1\right) \cdot f\left(s_{i}\right) \geq f(R(v))
$$

Recalling that $f(R(w))=\sum_{s_{i} \in R(w)} f\left(s_{i}\right)$ holds,

$$
\sum_{w \in W} f(R(w))=\sum_{w \in W} \sum_{s_{i} \in R(w)} f\left(s_{i}\right)
$$

Since $s_{i} \in R(w)$ is equivalent $w \in V_{i}$,

$$
\begin{aligned}
\sum_{w \in W} \sum_{s_{i} \in R(w)} f\left(s_{i}\right) & =\sum_{i \in\{1, \ldots, d\}} \sum_{w \in W \cap V_{i}} f\left(s_{i}\right) \\
& =\sum_{i \in I_{W}} \sum_{w \in W \cap V_{i}} f\left(s_{i}\right) \quad\left(\text { from } W \cap V_{i}=\emptyset \text { for } i \notin I_{W}\right) \\
& =\sum_{i \in I_{W}}\left|W \cap V_{i}\right| \cdot f\left(s_{i}\right) .
\end{aligned}
$$

Thus, the left hand side of (23) is equal to $\sum_{i \in I_{W}} f\left(s_{i}\right)$ from (24) and (25). Hence what remains is to prove that $\sum_{i \in I_{W}} f\left(s_{i}\right) \geq f(R(v))$ holds. To prove this inequality, it is sufficient to prove that $R(v) \subseteq\left\{s_{i}: i \in I_{W}\right\}$ holds. Since $s_{i} \in R(v)$ is equivalent to $v \in V_{i},\left|W \cap V_{i}\right| \neq \emptyset$ holds, i.e., $i \in I_{W}$. This completes the proof. 


\section{References}

[1] J. Edmonds. Submodular functions, matroids, and certain polyhedra. In R. Guy, H. Hanani, N. Sauer, and J. Schönheim, editors, Combinatorial Structures and their Applications, pages 69-87. Gordon and Breach, New York, 1970.

[2] J. Edmonds. Edge-disjoint branchings. In R. Rustin, editor, Combinatorial Algorithms, pages 91-96. Academic Press, New York, 1973.

[3] H. N. Gabow. A matroid approach to finding edge connectivity and packing arborescences. Journal of Computer and System Sciences, 50(2):259-273, 1995.

[4] N. Kamiyama, N. Katoh, and A. Takizawa. An efficient algorithm for the evacuation problem in a certain class of a network with uniform path-lengths. In Proceedings of the third International Conference on Algorithmic Aspects in Information and Management, volume 4508 of Lecture Notes in Computer Science, pages 178-190. Springer, June 2007.

[5] L. Lovász. On two minimax theorems in graph. J. Comb. Theory, Ser. B, 21(2):96-103, 1976.

[6] A. Schrijver. Combinatorial Optimization: Polyhedra and Efficiency. Springer, 2003.

[7] P. Tong and E. L. Lawler. A faster algorithm for finding edge-disjoint branchings. Inf. Process. Lett., 17(2):73-76, 1983. 